OPEN ACCESS

Edited by:

Vera Hermina Koch,

University of São Paulo, Brazi

Reviewed by:

Lyndsay Harshman,

The University of lowa, United States

Francisco Cano,

University of Chile, Chile

*Correspondence:

Ekaterini Siomou

eksiomou@yahoo.gr

Specialty section: This article was submitted to

Pediatric Nephrology

a section of the journa

Frontiers in Pediatrics

Received: 29 April 2021

Accepted: 19 July 2021

Published: 12 August 2021

Citation:

Karava V, Kondou A, Dotis J, Christoforidis A, Taparkou A, Tsioni K,

Farmaki E, Kollios K, Siomou E,

Liakopoulos V and Printza N (2021) Association Between Secondary

Hyperparathyroidism and Body

Composition in Pediatric Patients With Moderate and Advanced Chronic

Kidney Disease.

Front. Pediatr. 9:702778

doi: 10.3389/fped.2021.702778

\section{Association Between Secondary Hyperparathyroidism and Body Composition in Pediatric Patients With Moderate and Advanced Chronic Kidney Disease}

\author{
Vasiliki Karava ${ }^{1}$, Antonia Kondou ${ }^{1}$, John Dotis ${ }^{1}$, Athanasios Christoforidis ${ }^{2}$, \\ Anna Taparkou ${ }^{3}$, Konstantina Tsioni ${ }^{4}$, Evangelia Farmaki ${ }^{3}$, Konstantinos Kollios ${ }^{5}$, \\ Ekaterini Siomou ${ }^{6 *}$, Vassilios Liakopoulos ${ }^{7}$ and Nikoleta Printza ${ }^{1}$ \\ ${ }^{1}$ Pediatric Nephrology Unit, First Department of Pediatrics, Hippokratio General Hospital, Aristotle University of Thessaloniki, \\ Thessaloniki, Greece, ${ }^{2}$ Pediatric Endocrinology Unit, First Department of Pediatrics, Hippokratio General Hospital, Aristotle \\ University of Thessaloniki, Thessaloniki, Greece, ${ }^{3}$ First Department of Paediatrics, Pediatric Immunology and Rheumatology \\ Referral Center, Hippokratio General Hospital, Aristotle University of Thessaloniki, Thessaloniki, Greece, ${ }^{4}$ Biopathology \\ Laboratory, Hippokratio General Hospital, Thessaloniki, Greece, ${ }^{5}$ Third Department of Pediatrics, Hippokratio General \\ Hospital, Aristotle University of Thessaloniki, Thessaloniki, Greece, ${ }^{6}$ Department of Pediatrics, University Hospital of loannina, \\ Ioannina, Greece, ' Division of Nephrology and Hypertension, First Department of Internal Medicine, AHEPA Hospital, \\ Aristotle University of Thessaloniki, Thessaloniki, Greece
}

Objective: This single center cross-sectional study aims to investigate the association between secondary hyperparathyroidism and body composition in pediatric patients with moderate (stage 3) and advanced (stage 4-5) chronic kidney disease (CKD).

Methods: 61 patients (median age: 13.4 years) were included. Body composition indices, including lean tissue index (LTI) and fat tissue index (FTI), were measured using multi-frequency bio-impedance spectroscopy. Muscle wasting was defined as LTI adjusted to height-age $(\mathrm{HA}) \mathrm{z}$-score $<-1.65 \mathrm{SD}$ and high adiposity as $\mathrm{FTI} \mathrm{z}$-score $>1.65$ SD. Serum mineral metabolism parameters, including serum intact parathormone (iPTH), calcium, phosphorus and 25-hydroxyvitamin D, as well as serum leptin were measured in each patient. In advanced CKD patients, the mean values of serum mineral laboratory parameters of the 6 months prior to body composition assessment were recorded, and alfacalcidol index, defined as weekly alfacalcidol dose (mcg/week) per pg/ml of iPTH $\times$ 1,000 , was calculated.

Results: In moderate CKD (31 patients), high iPTH (>90 ng/ml) was observed in 10 (32.3\%) patients and was associated with higher FTI z-score $(p=0.022)$. Moreover, serum $\mathrm{iPTH}$ was negatively correlated to LTI HA z-score ( $r$ s $=-0.486, p=0.006$ ), and positively correlated to serum leptin levels ( $r s=0.369, p=0.041$ ). The positive correlation between FTI z-score and iPTH ( $r s=0.393, p=0.039$ ) lost significance after adjustment for serum leptin. IPTH was positively associated with high adiposity (12 patients, 38.7\%) after adjustment for the other mineral metabolism parameters (OR 1.023, 95\% Cl 1.002-1.045, $p=0.028$ ). In advanced CKD (30 patients), no significant correlation was observed between $\mathrm{PTTH}$ and body composition indices and serum leptin 
levels. Eleven (36.7\%) patients with muscle wasting presented lower alfacalcidol index $(p=0.017)$. Alfacalcidol index $\leq 24$ was strongly associated with muscle wasting after adjustment for CKD stage and other mineral metabolism parameters (OR 7.226, 95\% Cl 1.150-45.384, $p=0.035$ ).

Conclusion: Secondary hyperparathyroidism is associated with high adiposity in moderate but not in advanced CKD, with leptin acting as a potential contributive factor. In advanced CKD, targeting higher alfacalcidol weekly dose per each unit of serum PTH seems beneficial for preventing muscle wasting.

Keywords: secondary hyperparathyroidism, parathormone, muscle wasting, fat, leptin, chronic kidney disease, alfacalcidol, children

\section{INTRODUCTION}

Chronic kidney disease (CKD) is generally considered as a chronic catabolic disease, resulting in increased muscle protein and fat metabolic rate. Muscle wasting, involving loss of muscle mass and/or muscle strength, is present even in the early course of the disease, while its prevalence rises with decline of kidney function, ultimately affecting more than $40 \%$ of pediatric patients with advanced CKD $(1,2)$. Multiple mechanisms have been implicated in its pathogenesis, including anemia, chronic metabolic acidosis, anorexia, increased oxidative stress, accumulation of inflammatory cytokines and endocrine disorders, such as disturbed growth hormone/insulin growth factor-1 signaling (3). Fat loss is less frequently marked in pediatric patients and is predominantly encountered in advanced CKD. Additionally, increased adiposity, primarily attributed to reduced physical activity and adoption of high-fat western diet, is increasingly observed in pediatric CKD patients, with a prevalence of overweight and obesity among European pediatric patients on renal replacement therapy of 20.8 and $12.5 \%$, respectively (4). Both muscle wasting and high adiposity, which may be coupled especially in case of normal-weight obesity, have been proven unfavorable prognostic factors for the patient overall morbidity. Muscle wasting eventually leads to protein energy wasting and frailty phenotype, which may compromise patient life quality, deteriorate bone disease $(5,6)$ and increase hospitalization (7) and mortality risk (8), while high body adiposity levels have been associated with aggravated metabolic and cardiovascular risk (9-11), more rapid CKD progression (12) and reduced access to kidney transplantation (13). Therefore, early recognition of body composition disturbances and prompt management of the related pathogenetic risk factors as well as the subsequent adverse patient outcomes are crucial for the optimal healthcare delivery of these patients.

Nowadays, increasing evidence suggest that fat and muscle tissue are considered as endocrine organs, while adipokines and myokines facilitate their pleiotropic endocrine activity. Among the interactions between fat and muscle tissue and various metabolic pathways, results from experimental and clinical studies indicate a direct link between serum parathyroid hormone (PTH) and fat and muscle metabolism and a possible contributive role of adipocyte derived leptin on the crosstalk between PTH and fat status. It is current knowledge that obesity is commonly linked to higher serum PTH levels, in both general adult (14) and pediatric population (15), ascertaining a direct endocrine crosstalk between adipose tissue and parathyroid gland. This finding was also confirmed in CKD patients, where secondary hyperparathyroidism (SHPT) was more frequently observed in obese pre-dialysis adult patients $(16,17)$. Similar data in pediatric population are limited to kidney transplant patients, in whom high body mass index (BMI) was positively associated with SHPT in a recent cohort study (18). Besides, lower serum PTH levels in advanced CKD adult patients may reflect poor nutritional status, which in turn, increases mortality risk (19, 20). Nevertheless, in vivo studies in CKD mice have recently pointed out that SHPT may promote adipose tissue browning and muscle wasting, through stimulation of thermogenic gene expression, and more precisely of uncoupling protein-1 (Ucp1), ultimately leading to cachexia $(21,22)$. Data from clinical studies investigating the possible lipolytic and muscle proteolytic effects of high PTH are lacking and primarily concern adult population, where severe SHPT negatively affects body adiposity levels in CKD patients requiring parathyroidectomy $(23,24)$. The aim of this study was to investigate the association between body composition, involving both body fat and muscle status, using multi-frequency bioimpedance spectroscopy (BIS) technique, and serum leptin with serum PTH status in both moderate and advanced CKD pediatric patients.

\section{MATERIALS AND METHODS}

We conducted a cross-sectional study on the data of children and adolescents with CKD followed-up at the 1st and 3rd Department of Pediatrics at the Hippokratio General Hospital of Thessaloniki from March 2018 to December 2020. Inclusion criteria included: (i) age of participants between 5 and 19 years old and (ii) estimated glomerular filtration rate (eGFR) $<60 \mathrm{ml} / \mathrm{min} / 1.73$ $\mathrm{m}^{2}$, as calculated by the revised Schwartz formula. Moderate CKD was defined as the presence of CKD stage 3 and advanced CKD as the presence of CKD stage 4 to 5 , including patients on chronic dialysis (CKD 5D).

Anthropometric data, including weight, height and body mass index (BMI), were converted into $z$-scores based on Centers for Disease and Control and Prevention (CDC) reference values for healthy children of the same age and sex (25). BMI 
$\mathrm{z}$-score adjusted to height age (BMI HA z-score) was calculated for all patients. Patient body composition was estimated by multi-frequency BIS technique, using the same portable BIS device (Body composition monitor, BCM, Fresenius Medical Care, Bad Homburg, Germany). Both anthropometric and body composition measurements were effectuated on the same day by the same physician. Participants with CKD 3-4 were instructed to refrain from eating and drinking for at least $8 \mathrm{~h}$ prior to testing, while body composition assessment was obtained at the estimated dry weight in CKD 5D patients. In detail, BIS measurement was carried out $3 \mathrm{~h}$ post dialysis session and after drainage of peritoneal fluid in patients on peritoneal dialysis, and $1 \mathrm{~h}$ after the end of a mid-week hemodialysis session in patients on hemodialysis. Muscle mass and fat mass status were estimated

TABLE 1 | Distribution of anthropometric parameters, body composition indices and laboratory data in chronic kidney disease (CKD) stage 3 patients with and without high $\mathrm{PTH}(>90 \mathrm{ng} / \mathrm{ml})$ and in CKD stage 4-5D patients.

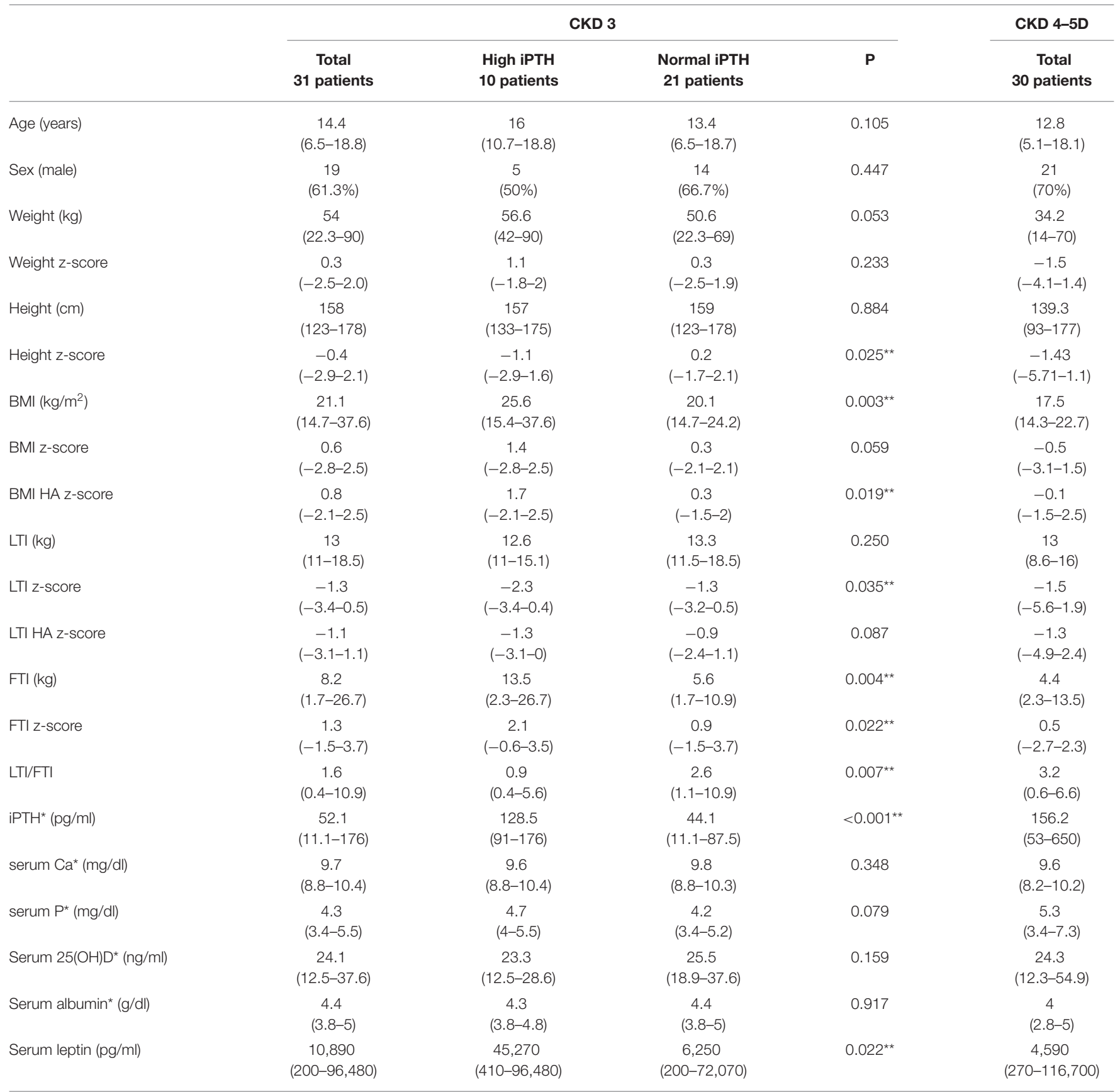

BMI, body mass index; LTI, lean tissue index; HA, height-age; FTI, fat tissue index; iPTH, intact parathormone; Ca, calcium; P, phosphorus; 25(OH)D, 25-hydroxyvitamin D. ${ }^{*}$ In patients with CKD stage 4,5D the 6-month mean serum $\mathrm{Ca}, P, 25(\mathrm{OH}) \mathrm{D}$ and albumin were recorded.

${ }^{* *} p$ statistically significant. 
using lean tissue index (LTI) and fat tissue index (FTI), defined as lean mass to height ${ }^{2}\left(\mathrm{~kg} / \mathrm{m}^{2}\right)$ and fat mass to height ${ }^{2}\left(\mathrm{~kg} / \mathrm{m}^{2}\right)$, respectively, which were derived from the BIS device impedance data. LTI to FTI ratio (LTI/FTI) was also calculated for each patient. All LTI and FTI values were converted into z-scores using body composition reference curves for healthy children in the United Kingdom (UK) (26). Muscle wasting and reduced adiposity were defined as the presence of LTI adjusted to height age z-score (LTI HA z-score) and FTI z-score lower than the 5th percentile ( $\mathrm{z}$-score $<-1.65 \mathrm{SD}$ ), while high LTI and increased adiposity as the presence of LTI adjusted to height age $\mathrm{z}$-score (HA) (LTI HA) and FTI z-score higher than the 95th percentile (z-score $>1.65 \mathrm{SD})$.

Serum mineral metabolism parameters, including serum intact parathormone (iPTH), calcium (Ca), phosphorus (P) and 25-hydroxyvitamin D $[25(\mathrm{OH}) \mathrm{D}]$, which were analyzed at the same local hospital laboratory using standard commercial assays, were collected for all patients on the day of body composition assessment. In moderate CKD, high iPTH was defined as iPTH $>90 \mathrm{ng} / \mathrm{ml}$, based on local laboratory reference ranges and the highest tercile of iPTH values in this patient group. In patients with advanced $\mathrm{CKD}$, mean values of serum mineral metabolism parameters were recorded for a 6-month period prior to body composition assessment. All patients with advanced CKD had a minimum of 3 measurements of serum mineral metabolism parameters during the study period. Alfacalcidol treatment was administered in all patients with advanced CKD, in form of drops or capsules, while none of the patient with moderate CKD was treated. The 6-month mean daily alfacalcidol dose was calculated. Moreover, alfacalcidol index, defined as 6-month mean alfacalcidol dose (mcg/week) per pg/ml of iPTH $\times 1,000$, was calculated in advanced CKD patients. Of note, none of the patients was under calcimimetic treatment during the study period. Finally, serum leptin levels, using ELISA technique (R\&D systems, Minneapolis, MN, USA) was measured in all patients on the day of body composition assessment.

Data were expressed as median values and ranges. All statistical tests were performed using IBM corp. SPSS Statistics ${ }^{\circledR}$ software for Windows. Mann Whitney and Fisher's exact and multiple chi-square tests were used to compare the distribution of the on-study continuous and categorical parameters between different patient groups. Spearman correlation tests were used to assess the univariate correlations between the on-study study parameters, whereas spearman partial correlation analysis was used to assess the correlations between the on-study variables after adjustment for possible confounders. Univariate and backward multiple logistic regression were performed in order to detect association between mineral metabolism profile and increased adiposity in moderate CKD and muscle wasting in advanced CKD. A $p$-value of $<0.05$ was considered statistically significant.

\section{RESULTS}

Sixty-one patients, 40 males and 21 females, with a median age of 13.4 years (range 5.2-19.7), were included in this study. Etiology of CKD included congenital abnormalities of kidney and urinary tract in 39 (63.9\%), ciliopathy in 6
(9.8\%), hemolytic uremic syndrome in 5 (8.2\%), focal segmental glomerulosclerosis in $3(5 \%)$, congenital nephrotic syndrome in $2(3.3 \%)$, chronic interstitial nephritis in $1(1.6 \%)$, familial hyperuricemic nephropathy in $1(1.6 \%)$ and of unknown origin in $4(6.6 \%)$ patients, respectively. CKD stage 3 was present in 31 (50.8\%) patients, stage 4 in $13(21.3 \%)$ patients, while $17(27.9 \%)$ patients were on chronic dialysis; 14 on peritoneal dialysis and 3 on hemodialysis. Of note, 8 patients were previously kidney transplanted with a median duration from kidney transplantation of $7.1(5.7-8.9)$ years.

Patients were divided according to CKD stage, in those presenting moderate CKD (stage 3, 31 patients) and advanced CKD (stages 4 and 5D, 30 patients). Distribution of anthropometric parameters, body composition indices and laboratory data in patients with moderate and advanced CKD are illustrated in Table 1. In total, LTI HA z-score was within normal range in $24(77.4 \%)$ patients with moderate and in 18 (60.0\%) patients with advanced CKD, while low LTI HA z-score $(<-1.65 \mathrm{SD})$ was observed in $7(22.6 \%)$ patients with moderate and in $11(36.7 \%)$ patients with advanced CKD (Figure 1). High FTI z-score (>1.65 SD) was observed in 12 (38.7\%) patients with moderate and in $4(13.3 \%)$ patients with advanced CKD. Of note, low FTI z-score $(<-1.65$ SD) was observed in only $3(10 \%)$ patients with advanced CKD (Figure 1).

Among the patients with moderate CKD, patients with serum iPTH $>90 \mathrm{pg} / \mathrm{ml}(10,32.3 \%)$ presented lower height and LTI z-scores $(p=0.025, p=0.035$, respectively), higher BMI HA and FTI z-scores $(p=0.019, p=0.022$, respectively) and lower LTI/FTI ( $p=0.007$ ) (Table 1). Accordingly, in spearman correlation analysis, serum iPTH was negatively correlated to height and LTI-z-scores ( $\mathrm{rs}=-0.397, p=0.027$ and $\mathrm{rs}=$ $-0.416, p=0.020$, respectively), positively correlated to FTI $\mathrm{z}$-score ( $\mathrm{rs}=0.393, p=0.039$ ), and negatively correlated to LTI/FTI ( $\mathrm{rs}=-0.450, p=0.011$ ) (Table 2). As expected, serum iPTH was positively correlated to serum P levels ( $r s=0.465$, $p=0.008$ ), which were higher in patients with serum iPTH $>90 \mathrm{pg} / \mathrm{ml}$, although the association did not reach statistical significance $(p=0.079)$ (Tables 1,2$)$. Moreover, serum leptin was positively associated with serum iPTH $>90 \mathrm{pg} / \mathrm{ml}(p=$ 0.022 ) and positively correlated to serum iPTH levels ( $\mathrm{rs}=$ $0.369, p=0.041$ ). In mediation analysis, serum leptin was also positively correlated to FTI $\mathrm{z}$-score ( $\mathrm{rs}=0.738, p<$ 0.001 ), while the correlation between FTI z-score and serum iPTH lost significance after adjustment for serum leptin ( $\mathrm{rs}=$ $0.160, p=0.398$ ) (Figure 2). In univariate logistic regression analysis, serum iPTH was positively associated with high FTI zscore (>1.65 SD) (OR 1.024, 95\% CI 1.004-1.044, $p=0.018$ ) (Table 3) and in backward multiple logistic regression analysis the association remained significant after adjustment for other mineral metabolism laboratory parameters, including serum $\mathrm{Ca}$, $\mathrm{P}$ and 25(OH)D (OR 1.023, 95\% CI 1.002-1.045, $p=0.028)$.

In patients with advanced $\mathrm{CKD}, 6$-month mean serum iPTH was, as expected, significantly higher than serum iPTH in moderate CKD $(p<0.001)$, but no correlation was observed between serum iPTH and anthropometric parameters and body composition indices (Table 2). Moreover, serum iPTH was inversely but not significantly correlated to serum leptin ( $r$ $=-0.228, p=0.225$ ). Of note, serum leptin was positively 


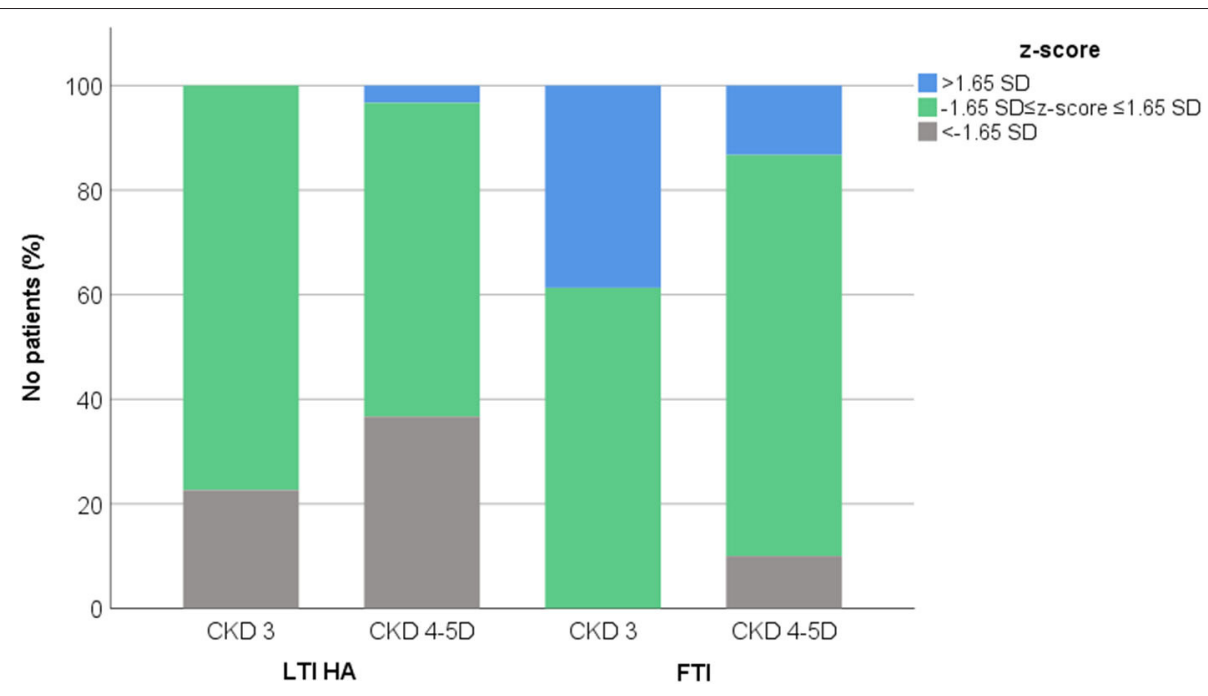

FIGURE 1 | Distribution of lean tissue index adjusted to height-age (LTI HA) and fat tissue index (FTI) Z-scores in chronic kidney disease (CKD) stage 3 and 4-5D patients.

TABLE 2 | Spearman correlation analysis of anthropometric parameters, body composition indices and laboratory data with serum intact parathormone (iPTH) in chronic kidney disease (CKD) stage 3 patients and with 6-month mean PPTH and alfacalcidol index [(mcg/week per pg/ml of PTH) $\times 1,000]$ in CKD stage 4,5D patients.

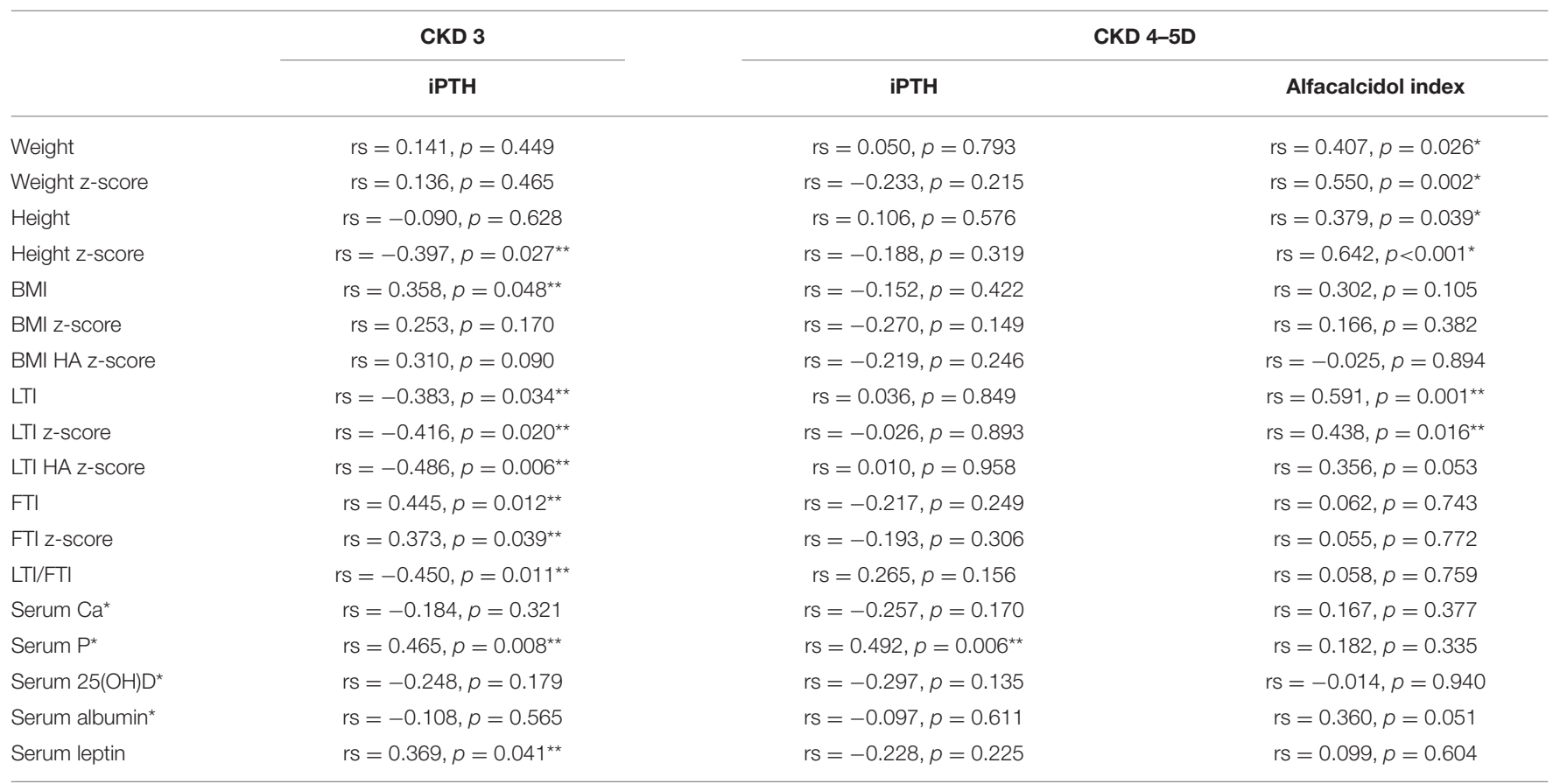

BMI, body mass index; LTI, lean tissue index; HA, height-age; FTI, fat tissue index; Ca, calcium; P, phosphorus; 25(OH)D, 25-hydroxyvitamin D. *In patients with CKD stage 4-5D the 6-month mean serum $\mathrm{Ca}, \mathrm{P}, 25(\mathrm{OH}) \mathrm{D}$ and albumin were recorded.

${ }^{* *} p$ statistically significant. 


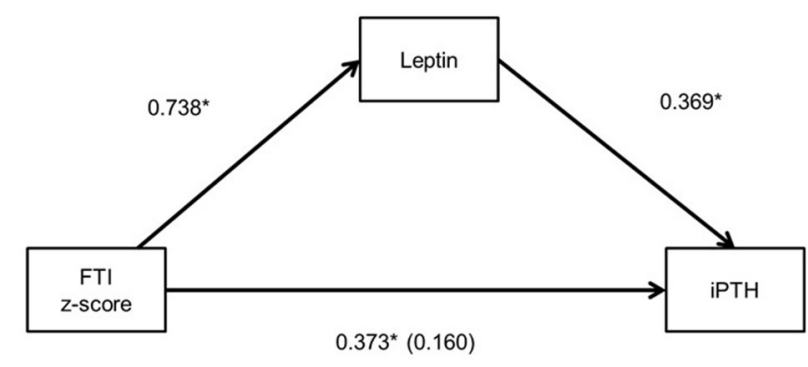

${ }^{\star}:$ p statistically significant

FIGURE 2 | Mediation analysis illustrating the spearman's correlation coefficient between serum leptin, intact parathormone (iPTH) levels and fat tissue index (FTI) Z-score and the partial spearman's correlation efficient (in parenthesis) between iPTH and FTI z-score after adjustment for serum leptin in chronic kidney disease (CKD) stage 3 patients.

correlated to FTI z-score ( $\mathrm{rs}=0.401, p=0.028$ ), and did not significantly differ from that in moderate CKD $(p=0.292)$.

In advanced $\mathrm{CKD}$, alfacalcidol therapy, with a median 6month mean daily dose of $0.5 \mathrm{mcg}$ (range $0.2-2 \mathrm{mcg}$ ), was adapted to patient serum iPTH levels ( $r s=0.876, p<0.001$ ) (Figure 3). Alfacalcidol index (median value 23.5, range 14.854.7) was positively correlated to weight, height and LTI z-score ( $\mathrm{rs}=0.550, p=0.002, \mathrm{rs}=0.642, p<0.001$ and $\mathrm{rs}=0.438$, $p=0.016$, respectively) (Table 2 ) and was significantly lower in patients with low LTI HA $z$-score $(<-1.65 \mathrm{SD})(p=0.017)$ and 6 -month mean serum albumin $<3.5 \mathrm{~g} / \mathrm{dl}$ (8 patients, 26.7\%) ( $p$ $=0.046$ ) (Figure 4). In further analysis, patients were divided according to approximate median alfacalcidol index in those with alfacalcidol index $>24$ (14 patients) and alfacalcidol index $\leq$ 24 (16 patients). The patient group with low alfacalcidol index was also stratified based on 6-month mean serum iPTH level (cut off $150 \mathrm{ng} / \mathrm{ml}$, median value). We observed that low LTI HA z-score was more prevalent in patients with alfacalcidol index $\leq 24$ and mean serum iPTH $\leq 150 \mathrm{ng} / \mathrm{ml}$ (5 patients, $71.4 \%$ ) or mean serum iPTH $>150 \mathrm{ng} / \mathrm{ml}$ (4 patients, $44.4 \%$ ), compared to those with alfacalcidol index $>24$ (2 patients, $14.3 \%)(p=0.032)$ (Figure 5). Alfacalcidol index $\leq 24$ was strongly associated with low LTI HA z-score (OR 7.714, 95\% CI $1.284-46.364, p=0.026)$ and the association remained significant in backward logistic regression analysis after adjustment for CKD stage and serum mineral metabolism parameters, including serum Ca, P and 25(OH)D (OR 7.226, 95\% CI 1.150-45.384, $p$ $=0.035$ ).

\section{DISCUSSION}

Pediatric CKD is commonly complicated by mineral and bone disorder (CKD-MBD), which mainly involves abnormal mineralization and disturbed bone turnover $(27,28)$. SHPT is the principal laboratory parameter of CKD-MBD, which is highly prevalent in advanced stages, but it may also occur earlier in the course of the disease. In our study, $32.3 \%$ of patients with moderate and $100 \%$ of patients with advanced CKD presented high serum iPTH levels. Additional to glomerular filtration rate deterioration, disturbed calcium, phosphorus and vitamin D balance constitute the principal risk factor of SHPT. As expected, in the current study, serum iPTH levels were significantly correlated to serum $\mathrm{P}$ in patients with either moderate or advanced CKD. Apart from controlling bone health, mineral homeostasis is involved in the regulation of various physiological processes, including renin angiotensin system (29), cardiovascular function (30), and bone hematopoiesis (31), while the interaction between mineral imbalance and other body system functions in the setting of CKD has recently gained research interest. Our study focuses on exploring the crosstalk between SHPT and body muscle and fat status in children with moderate and advanced CKD.

Body composition assessment is crucial for nutritional evaluation of pediatric patients with CKD. In our study, we applied BIS technique for estimation of body composition, and we used LTI-HA and FTI as indices of body muscle and fat status. We observed that prevalence of muscle wasting increased, while prevalence of high adiposity reduced from moderate to advanced CKD. Moreover, low adiposity, the prevalence of which is in general undetermined in pediatric CKD, was present in $10 \%$ of patients with advanced CKD.

In the current study, we found that, in moderate CKD, patients with SHPT presented higher FTI z-score levels, serum iPTH was positively correlated to FTI z-score and was independently associated with high adiposity. Although, obesity was associated with a higher risk of SHPT in pre-dialysis CKD adult patients (16, 17), to our knowledge, this association has not been described yet in pediatric population. Multiple pathogenetic mechanisms may explain this association. It is widely known that serum $25(\mathrm{OH}) \mathrm{D}$ levels are reduced in case of obesity in both adult and pediatric population $(32,33)$, primarily because of vitamin $\mathrm{D}$ sequestration or volumetric dilution in large body fat compartments, leading to decreased bioavailability from cutaneous and dietary sources $(34,35)$. In addition, sedentary lifestyle of subjects with high body adiposity levels, involving reduced sun ultraviolet B exposure, limited outdoor activities and inadequate mineral intake from unhealthy high caloric food, may aggravate hypovitaminosis D in this population (36). In pre-dialysis CKD, hypovitaminosis $\mathrm{D}$ is more prevalent in overweight patients in both adult and pediatric patients $(37,38)$. In our study, as expected, patients with SHPT presented lower serum 25(OH)D levels $(p=0.159)$, serum $\mathrm{iPTH}$ was negatively correlated to serum $25(\mathrm{OH}) \mathrm{D}$ levels (rs $=-0.248, p=0.179$ ), and serum $25(\mathrm{OH}) \mathrm{D}$ levels were lower in patients with high FTI z-score level $(p=0.141)$, but none of these correlations reached statistical significance. Besides, the association between serum IPTH and high adiposity remained significant after adjustment for other serum mineral metabolism parameters, suggesting a direct crosstalk between adipose tissue and parathyroid gland. Experimental in vivo and in vitro studies have evocated that adipose tissue derived leptin stimulates PTH excretion either indirectly, through upregulation of bone fibroblast growth-factor 23 expression in parallel with downregulation of kidney calcitriol production, or even by direct stimulatory action of PTH secretion from parathyroid gland $(39,40)$. In clinical studies, serum leptin levels were positively 
TABLE 3 | Mann-Whitney and univariate logistic regression analysis to identify association of mineral metabolism laboratory parameters with high fat tissue index (FTI) z-score (12 patients) in chronic kidney disease (CKD) stage 3 patients.

\begin{tabular}{|c|c|c|c|c|c|}
\hline & High FTI z-score 12 patients & Normal FTI z-score 19 patients & $p$ & OR (95\% Cl) & $p$ \\
\hline iPTH & $97.2(44-176)$ & $38(11-173)$ & $p=0.001^{\star}$ & 1.024 (1.004-1.044) & $p=0.018^{\star}$ \\
\hline Serum Ca & 9.7 (8.8-10.4) & 9.7 (8.8-10.3) & $p=0.952$ & $1.085(0.209-5.646)$ & $p=0.922$ \\
\hline Serum P & $4.7(3.5-5.5)$ & $4.2(3.4-5.4)$ & $p=0.152$ & $2.600(0.669-10.107)$ & $p=0.168$ \\
\hline Serum 25(OH)D & 23.7 (18.2-28.6) & $26(12.5-37.6)$ & $p=0.141$ & 0.903 (0.762-1.069) & $p=0.235$ \\
\hline
\end{tabular}

iPTH, intact parathormone; Ca, calcium; $P$, phosphorus; $25(\mathrm{OH}) D, 25$-hydroxyvitamin D.

* $p$ statistically significant.

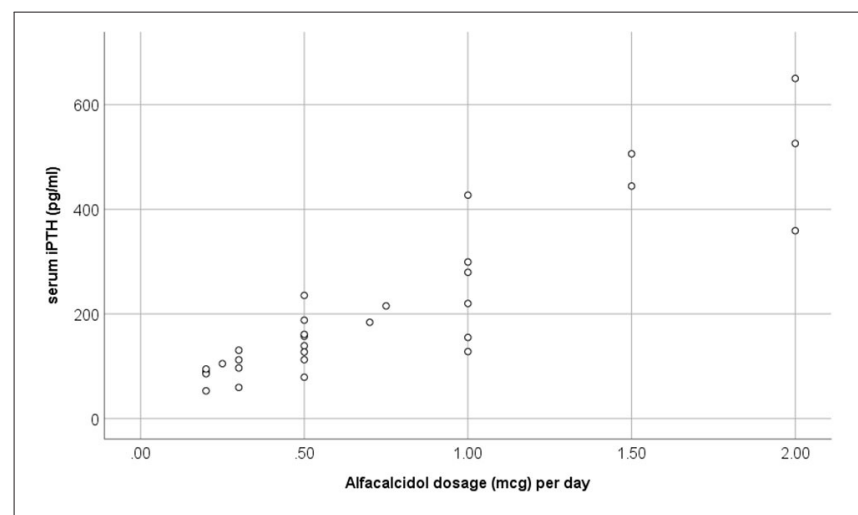

FIGURE 3 | Scatter plot analysis of 6-month mean alfacalcidol dosage (mcg) per day and mean serum intact parathormone (iPTH) in chronic kidney disease (CKD) stage 4-5D patients.

correlated to serum PTH levels in both general obese adult and pediatric population $(41,42)$. In our study, we remarked a broad range of serum leptin levels which accords with the large variety of FTI values observed in our population. As expected, serum leptin levels were positively FTI z-score. Moreover, serum leptin levels were higher in patients with SHPT and were positively correlated to serum iPTH, while the correlation between serum iPTH and FTI z-score lost significance after adjustment for serum leptin. These findings may suggest that high adiposity could enhance the risk of SHPT occurrence in moderate $\mathrm{CKD}$ and serum leptin may contribute to their association. Further large-scale studies are needed to confirm these results.

The association between serum leptin and SHPT has been explored in adult CKD population but the results are inconsistent among different studies (43), where an inverse (44), positive (45) or absence of correlation (46) between the two laboratory parameters was evocated. Moreover, according to an in-vitro study on differentiated adipocytes from humans with severe SHPT, high PTH levels suppressed adipocyte leptin production via inhibition of Akt signaling, indicating a negative effect of $\mathrm{PTH}$ on adipocyte leptin secretion (25). In our study, although serum iPTH was positively correlated to serum leptin in moderate CKD, an inverse non-statistically significant correlation between the two parameters was observed in advanced CKD patients. Of note, while serum leptin was positively correlated to FTI z-score, the

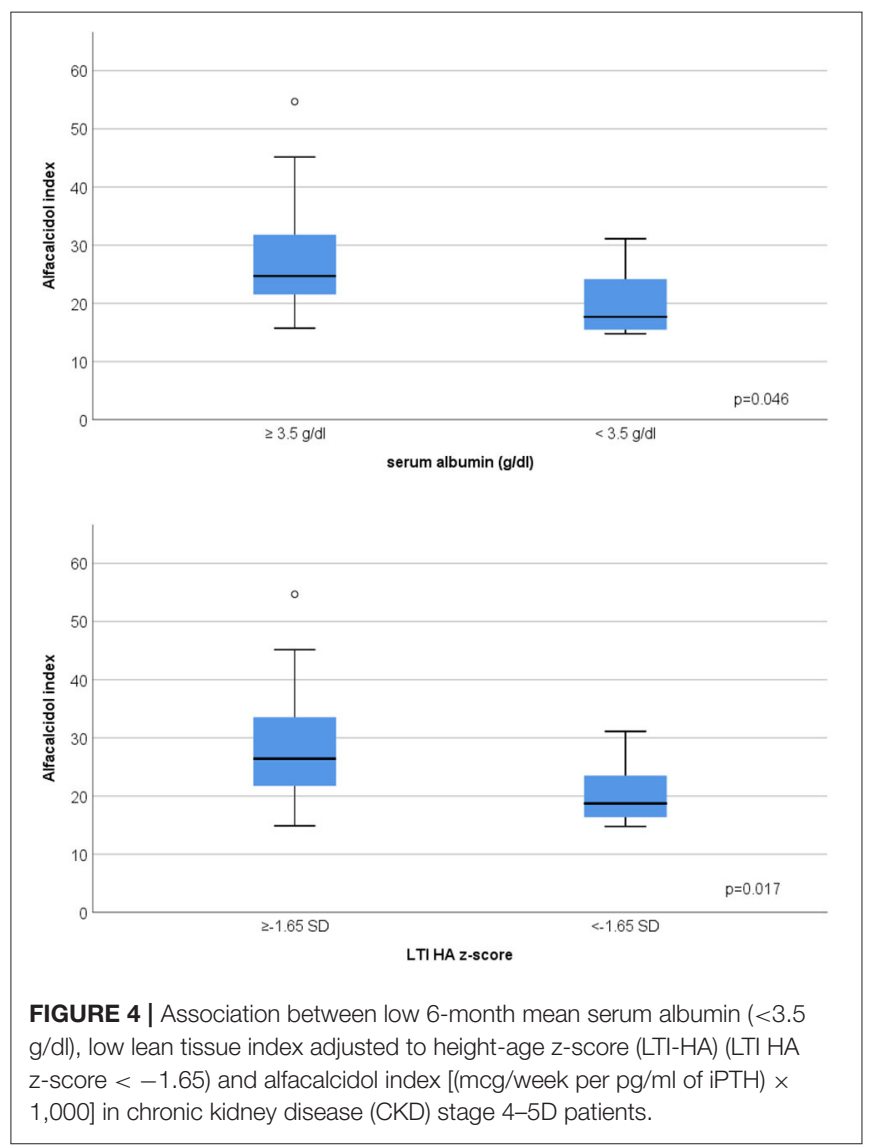

strength of correlation was attenuated in patients with advanced $\mathrm{CKD}$, suggesting that apart from fat mass, other factors may contribute to serum leptin expression in late CKD stages, such as inflammatory cytokine expression (47). Further studies are needed to explore the impact of PTH on serum leptin and the potential contributive role of inflammatory cytokine profile on their correlation in advanced CKD.

Few clinical adult studies have investigated the relation between serum PTH and body composition in advanced CKD. Most clinical studies suggest that serum PTH may serve as a nutritional marker, since it is positively correlated to both fat and muscle mass (48) especially in male population, it generally decreases along with decline of BMI (21), while patients with 


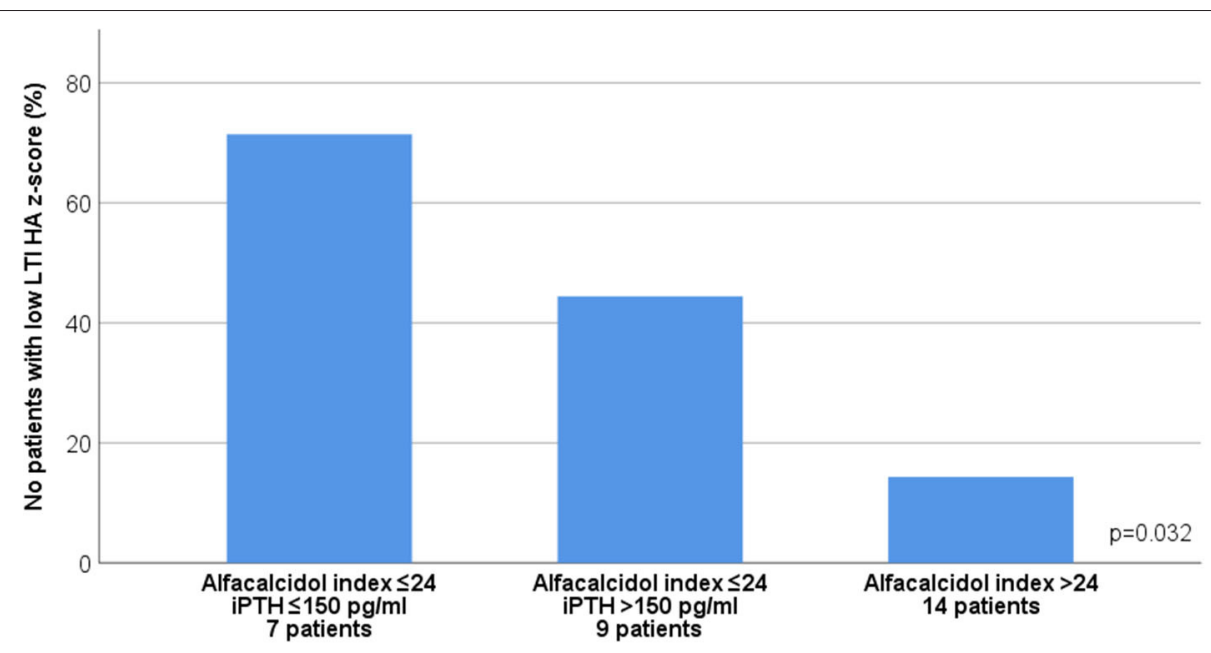

FIGURE 5 | Association between 6-month mean alfacalcidol index ([mcg/week per pg/ml of iPTH] $\times 1000$ ) and low lean tissue index adjusted to height-age z-score (LTI-HA) (LTI HA z-score < -1.65) after stratification by 6-month mean serum intact parathormone (iPTH) levels in chronic kidney disease (CKD) stages 4-5D patients.

serum PTH $<150 \mathrm{pg} / \mathrm{ml}$ present more frequently markers of protein-energy wasting and inflammation, involving reduced muscle mass and serum albumin levels, and generally receive lower active vitamin D analogs (20). In our study, no significant correlation was observed between 6-month mean serum iPTH and anthropometric parameters or body composition indices in patients with advanced CKD. Nevertheless, we found that the ratio of alfacalcidol weekly dose per each unit of serum iPTH, defined as alfacalcidol index, was lower in patients with muscle wasting and low serum albumin, while alfacalcidol index $\leq 24$ was significantly associated with muscle wasting after adjustment for other serum mineral metabolism parameters. Of note, no correlation was observed between alfacalcidol index and FTI. In adult patients on chronic hemodialysis, Shinaberger et al., mentioned that a higher weekly paricalcitol dosage per each unit of serum PTH was associated with a progressive greater survival (49). The benefits of a higher ratio of vitamin D receptor activator dosage to serum PTH in terms of patient nutritional status has never been previously explored. In our study, patients with lower alfacalcidol index consisted in those with lower serum iPTH levels and subsequently lower alfacalcidol dose and in those with higher serum iPTH levels inadequately controlled by alfacalcidol treatment. Both patient groups presented higher muscle wasting prevalence (71.4 and $44.4 \%$, respectively) compared to those with higher alfacalcidol index $(14.3 \%)$. It is probable that in the first patient group, malnutrition is the main cause of lower alfacalcidol index, and therefore muscle wasting, in accordance with the findings from clinical adult studies previously described. In the second patient group, we speculate that the relatively high serum iPTH levels coupled with relatively low alfacalcidol dosage are responsible for the remarked reduced LTI. As already mentioned, SHPT has been recently incriminated for muscle wasting, according to the results of in-vivo studies $(22,23)$. Furthermore, vitamin $\mathrm{D}$ deficiency is generally considered as a contributor factor of muscle wasting in both general population and CKD (50) while native and active vitamin $\mathrm{D}$ supplementation seem to reduce adipose tissue browning and muscle wasting, by modulating the expression of thermogenic genes on a mouse model of CKD-associated cachexia (51). Therefore, we presume that both lower serum iPTH levels due to malnutrition and higher serum iPTH levels coupled with insufficient active vitamin D analog dosage are associated with poor muscle status in pediatric advanced CKD.

Optimal PTH control in advanced CKD remains a cornerstone challenge for pediatric nephrology in clinical practice. The benefits of active vitamin $\mathrm{D}$ analogs for reducing serum PTH levels are counterweighted by the risk of rising serum calcium levels and subsequently calcium-phosphate product, eventually leading to extraskeletal calcifications and worsening of cardiovascular disease. Calcimimetics and new oral phosphate binders, such as sucroferric oxyhydroxide, which are currently approved for administration in pediatric population, have been proven effective for serum phosphorus and PTH management $(52,53)$. The benefits of these drugs on the preservation of muscle mass in advanced pediatric CKD needs to be answered in the future.

Our study has some limitations. Firstly, the small number of included patients of various ages with a broad spectrum of CKD severity preclude us from making definite conclusions. Moreover, the limited number of recruited patients with CKD 4 and CKD 5 and 5D did not allow us to perform a further subgroup data analysis for each CKD stage patient groups. In addition, the impact of pubertal status on PTH values and therefore on the association between PTH and body composition indices was not taken into account in our study. Furthermore, adjustment of serum iPTH to alfacalcidol dosage without considering other medications, which may have also influenced serum iPTH levels, such as oral phosphate binders, calcimimetics, native vitamin $\mathrm{D}$ and calcium supplement, limits the strength of our results. 
Finally, the cross-sectional nature of the study does not allow us to safely determine the cause-effect relationships of the onstudy associations.

\section{CONCLUSION}

In conclusion, our study showed that pediatric patients with high adiposity may present secondary hyperparathyroidism earlier in the course of $\mathrm{CKD}$, suggesting a direct link between adipose tissue and parathyroid gland. The potential benefits of lowering high adiposity in preventing secondary hyperparathyroidism in children and adolescents with moderate CKD needs further investigation. Moreover, we observed that pediatric patients with advanced CKD who maintain low serum iPTH levels with low alfacalcidol dosage and those who preserve high serum iPTH despite active vitamin D analog therapy present higher frequency of muscle wasting, suggesting that alfacalcidol index may serve as a marker of evaluation of nutritional status in everyday clinical practice. Additional large-scale prospective studies are required to explore the prognostic value of alfacalcidol index on estimating the progression of muscle wasting in children under active vitamin D analogs.

\section{REFERENCES}

1. Abraham AG, Mak RH, Mitsnefes M, White C, Moxey-Mims M, Warady B, et al. Protein energy wasting in children with chronic kidney disease. Pediatr Nephrol. (2014) 29:1231-8. doi: 10.1007/s00467-014-2768-9

2. Oliveira EA, Cheung WW, Toma KG, Mak RH. Muscle wasting in chronic kidney disease. Pediatr Nephrol. (2018) 33:789-98. doi: 10.1007/s00467-017-3684-6

3. Mak RH, Cheung WW, Zhan JY, Shen Q, Foster BJ. Cachexia and proteinenergy wasting in children with chronic kidney disease. Pediatr Nephrol. (2012) 27:173-81. doi: 10.1007/s00467-011-1765-5

4. Bonthuis M, van Stralen KJ, Verrina E, Groothoff JW, Alonso Melgar Á, Edefonti A, et al. Underweight, overweight and obesity in paediatric dialysis and renal transplant patients. Nephrol Dial Transplant. (2013) 28 (Suppl. 4):iv195-iv204. doi: 10.1093/ndt/gft259

5. Karava V, Dotis J, Christoforidis A, Kondou A, Printza N. Muscle-bone axis in children with chronic kidney disease: current knowledge and future perspectives. Pediatr Nephrol. (2021). doi: 10.1007/s00467-021-04936-w. [Epub ahead of print].

6. Karava V, Dotis J, Christoforidis A, Liakopoulos V, Kondou A, Tsigaras G, et al. Association between insulin growth factor-1, bone mineral density, and frailty phenotype in children with chronic kidney disease. Pediatr Nephrol. (2021). doi: 10.1007/s00467-021-04918-y. [Epub ahead of print].

7. Sgambat K, Matheson MB, Hooper SR, Warady B, Furth S, Moudgil A. Prevalence and outcomes of fragility: a frailty-inflammation phenotype in children with chronic kidney disease. Pediatr Nephrol. (2019) 34:25639. doi: 10.1007/s00467-019-04313-8

8. Karava V, Dotis J, Kondou A, Christoforidis A, Liakopoulos V, Tsioni K, et al. Association between relative fat mass, uric acid, and insulin resistance in children with chronic kidney disease. Pediatr Nephrol. (2021) 36:42534. doi: $10.1007 /$ s00467-020-04716-y

9. Wong CS, Gipson DS, Gillen DL, Emerson S, Koepsell T, Sherrard DJ, et al. Anthropometric measures and risk of death in children with endstage renal disease. Am J Kidney Dis. (2000) 36:811-9. doi: 10.1053/ajkd.2000. 17674

10. Sgambat K, Roem J, Mitsnefes M, Portale AA, Furth S, Warady B, et al. Waist-to-height ratio, body mass index, and cardiovascular risk profile in

\section{DATA AVAILABILITY STATEMENT}

The original contributions presented in the study are included in the article/supplementary material, further inquiries can be directed to the corresponding author/s.

\section{ETHICS STATEMENT}

The studies involving human participants were reviewed and approved by Committee for Bioethics and Ethics of School of Medicine of Aristotle University of Thessaloniki. Written informed consent to participate in this study was provided by the participants' legal guardian/next of kin.

\section{AUTHOR CONTRIBUTIONS}

NP, AC, ES, and VK conceived and designed the analysis of the study. VK, AK, JD, and KK collected the data. AT and KT performed the laboratory tests. VL provided the BIS device. VK, $\mathrm{AK}$, and JD performed the statistical analysis. VK wrote the paper. AC, EF, ES, and VL reviewed the paper. NP made the final revision. All authors contributed to the article and approved the submitted version.

children with chronic kidney disease. Pediatr Nephrol. (2018) 33:157783. doi: $10.1007 /$ s00467-018-3987-2

11. Karava V, Printza N, Dotis J, Demertzi D, Antza C, Kotsis V, et al. Body composition and arterial stiffness in pediatric patients with chronic kidney disease. Pediatr Nephrol. (2019) 34:1253-60. doi: 10.1007/s00467-019-04224-8

12. Lalan S, Jiang S, Ng DK, Kupferman F, Warady BA, Furth S, et al. Cardiometabolic risk factors, metabolic syndrome, and chronic kidney disease progression in children. J Pediatr. (2018) 202:163-70. doi: 10.1016/j.jpeds.2018.06.007

13. Ku E, Glidden DV, Hsu CY, Portale AA, Grimes B, Johansen KL. Association of body mass index with patient-centered outcomes in children with ESRD. $J$ Am Soc Nephrol. (2016) 27:551-8. doi: 10.1681/ASN.2015010008

14. Kamycheva E, Sundsfjord J, Jorde R. Serum parathyroid hormone level is associated with body mass index. The 5th Tromsø study. Eur J Endocrinol. (2004) 151:167-72. doi: 10.1530/eje.0.1510167

15. Reinehr T, de Sousa G, Alexy U, Kersting M, Andler W. Vitamin D status and parathyroid hormone in obese children before and after weight loss. Eur J Endocrinol. (2007) 157:225-32. doi: 10.1530/EJE-07-0188

16. Kovesdy CP, Ahmadzadeh S, Anderson JE, Kalantar-Zadeh K. Obesity is associated with secondary hyperparathyroidism in men with moderate and severe chronic kidney disease. Clin J Am Soc Nephrol. (2007) 2:10249. doi: 10.2215/CJN.01970507

17. Saab G, Whaley-Connell A, McFarlane SI, Li S, Chen SC, Sowers JR, et al. Obesity is associated with increased parathyroid hormone levels independent of glomerular filtration rate in chronic kidney disease. Metabolism. (2010) 59:385-9. doi: 10.1016/j.metabol.2009.08.007

18. Vanderstraeten K, De Pauw R, Knops N, Bouts A, Cransberg K, El Amouri A, et al. Body mass index is associated with hyperparathyroidism in pediatric kidney transplant recipients. Pediatr Nephrol. (2021) 36:97786. doi: 10.1007/s00467-020-04796-w

19. Dukkipati R, Kovesdy CP, Colman S, Budoff MJ, Nissenson AR, Sprague SM, et al. Association of relatively low serum parathyroid hormone with malnutrition-inflammation complex and survival in maintenance hemodialysis patients. J Ren Nutr. (2010) 20:243-54. doi: 10.1053/j.jrn.2009.10.006

20. Drechsler C, Grootendorst DC, Boeschoten EW, Krediet RT, Wanner C, Dekker FW, et al. Changes in parathyroid hormone, body mass index and the 
association with mortality in dialysis patients. Nephrol Dial Transplant. (2011) 26:1340-6. doi: 10.1093/ndt/gfq541

21. Thomas SS, Mitch WE. Parathyroid hormone stimulates adipose tissue browning: a pathway to muscle wasting. Curr Opin Clin Nutr Metab Care. (2017) 20:153-7. doi: 10.1097/MCO.0000000000000357

22. Kir S, Komaba H, Garcia AP, Economopoulos KP, Liu W, Lanske B, et al. PTH/PTHrP receptor mediates cachexia in models of kidney failure and cancer. Cell Metab. (2016) 23:315-23. doi: 10.1016/j.cmet.2015.11.003

23. Peters BS, Jorgetti V, Martini LA. Body composition changes in haemodialysis patients with secondary hyperparathyroidism after parathyroidectomy measured by conventional and vector bioimpedance analysis. Br J Nutr. (2006) 95:353-7. doi: 10.1079/BJN20051637

24. Jiang Y, Zhang J, Yuan Y, Zha X, Xing C, Shen C, et al. Association of increased serum leptin with ameliorated anemia and malnutrition in stage 5 chronic kidney disease patients after parathyroidectomy. Sci Rep. (2016) 6:27918. doi: 10.1038/srep27918

25. Ogden CL, Kuczmarski RJ, Flegal KM, Mei Z, Guo S, Wei R, et al. Centers for disease control and prevention 2000 growth charts for the United States: improvements to the 1977 National Center for Health Statistics version. Pediatrics. (2002) 109:45-60. doi: 10.1542/peds.109.1.45

26. Wells JC, Williams JE, Chomtho S, Darch T, Grijalva-Eternod C, Kennedy K, et al. Body-composition reference data for simple and reference techniques and a 4-component model: a new UK reference child. Am J Clin Nutr. (2012) 96:1316-26. doi: 10.3945/ajcn.112.036970

27. Bakkaloglu SA, Wesseling-Perry K, Pereira RC, Wang HJ, Elashoff RM, Salusky IB. Value of the new bone classification system in pediatric renal osteodystrophy. Clin J Am Soc Nephrol. (2010) 5:1860-6. doi: 10.2215/CJN.01330210

28. Wesseling-Perry K, Pereira RC, Tseng CH, Elashoff R, Zaritsky JJ, Yadin O, et al. Early skeletal and biochemical alterations in pediatric chronic kidney disease. Clin J Am Soc Nephrol. (2012) 7:146-52. doi: 10.2215/CJN.05940611

29. Vaidya A, Brown JM, Williams JS. The renin-angiotensin-aldosterone system and calcium-regulatory hormones. J Hum Hypertens. (2015) 29:51521. doi: $10.1038 /$ jhh.2014.125

30. Voelkl J, Egli-Spichtig D, Alesutan I, Wagner CA. Inflammation: a putative link between phosphate metabolism and cardiovascular disease. Clin Sci. (2021) 135:201-27. doi: 10.1042/CS20190895

31. Tanaka M, Komaba H, Fukagawa M. Emerging association between parathyroid hormone and anemia in hemodialysis patients. Ther Apher Dial. (2018) 22:242-5. doi: 10.1111/1744-9987.12685

32. Pacifico L, Anania C, Osborn JF, Ferraro F, Bonci E, Olivero E, et al. Low 25(OH)D3 levels are associated with total adiposity, metabolic syndrome, and hypertension in Caucasian children and adolescents. Eur J Endocrinol. (2011) 165:603-11. doi: 10.1530/EJE-11-0545

33. Vimaleswaran KS, Berry DJ, Lu C, Tikkanen E, Pilz S, Hiraki LT, et al. Causal relationship between obesity and vitamin D status: bi-directional Mendelian randomization analysis of multiple cohorts. PLoS Med. (2013) 10:e1001383. doi: 10.1371/journal.pmed.1001383

34. Wortsman J, Matsuoka LY, Chen TC, Lu Z, Holick MF. Decreased bioavailability of vitamin D in obesity. Am J Clin Nutr. (2000) 72:6903. doi: $10.1093 /$ ajcn/72.3.690

35. Drincic AT, Armas LA, Van Diest EE, Heaney RP. Volumetric dilution, rather than sequestration best explains the low vitamin D status of obesity. Obesity. (2012) 20:1444-8. doi: 10.1038/oby.2011.404

36. Dong Y, Pollock N, Stallmann-Jorgensen IS, Gutin B, Lan L, Chen TC, et al. Low 25-hydroxyvitamin D levels in adolescents: race, season, adiposity, physical activity, and fitness. Pediatrics. (2010) 125:110411. doi: 10.1542/peds.2009-2055

37. Figuiredo-Dias V, Cuppari L, Garcia-Lopes MG, de Carvalho AB, Draibe SA, Kamimura MA. Risk factors for hypovitaminosis D in nondialyzed chronic kidney disease patients. J Ren Nutr. (2012) 22:4-11. doi: 10.1053/j.jrn.2011.02.001

38. Seeherunvong W, Abitbol CL, Chandar J, Zilleruelo G, Freundlich M. Vitamin D insufficiency and deficiency in children with early chronic kidney disease. $J$ Pediatr. (2009) 154:906-11.e1. doi: 10.1016/j.jpeds.2008.12.006

39. Tsuji K, Maeda T, Kawane T, Matsunuma A, Horiuchi N. Leptin stimulates fibroblast growth factor 23 expression in bone and suppresses renal 1alpha,25dihydroxyvitamin D3 synthesis in leptin-deficient mice. J Bone Miner Res. (2010) 25:1711-23. doi: 10.1002/jbmr.65
40. Lopez I, Pineda C, Raya AI, Rodriguez-Ortiz ME, Diaz-Tocados JM, Rios R. Leptin directly stimulates parathyroid hormone secretion. Endocrine. (2017) 56:675-8. doi: 10.1007/s12020-016-1207-z

41. Grethen E, Hill KM, Jones R, Cacucci BM, Gupta CE, Acton A. Serum leptin, parathyroid hormone, 1,25-dihydroxyvitamin $\mathrm{D}$, fibroblast growth factor 23 , bone alkaline phosphatase, and sclerostin relationships in obesity. J Clin Endocrinol Metab. (2012) 97:1655-62. doi: 10.1210/jc.2011-2280

42. Saber LM, Mahran HN, Baghdadi HH, Al Hawsawi ZM. Interrelationship between bone turnover markers, calciotropic hormones and leptin in obese Saudi children. Eur Rev Med Pharmacol Sci. (2015) 19:4332-43.

43. Polyzos SA, Duntas L, Bollerslev J. The intriguing connections of leptin to hyperparathyroidism. Endocrine. (2017) 57:37687. doi: 10.1007/s12020-017-1374-6

44. Kokot F, Chudek J, Karkoszka H, Adamczak M, Wiecek A, Klimek D. Does PTH influence leptin concentration in haemodialysed uraemic patients? Nephron. (1999) 82:372-3. doi: 10.1159/000045462

45. Kovesdy CP, Molnar MZ, Czira ME, Rudas A, Ujszaszi A, Rosivall L, et al. Associations between serum leptin level and bone turnover in kidney transplant recipients. Clin J Am Soc Nephrol. (2010) 5:2297304. doi: $10.2215 / \mathrm{CJN} .03520410$

46. Ghorban-Sabbagh M, Nazemian F, Naghibi M, Shakeri MT, AhmadiSimab S, Javidi-Dasht-Bayaz R. Correlation between serum leptin and bone mineral density in hemodialysis patients. J Renal Inj Prev. (2016) 5:1127. doi: 10.15171/jrip.2016.24

47. Pecoits-Filho R, Nordfors L, Heimbürger O, Lindholm B, Anderstam B, Marchlewska A, et al. Soluble leptin receptors and serum leptin in end-stage renal disease: relationship with inflammation and body composition. Eur $J$ Clin Invest. (2002) 32:811-7. doi: 10.1046/j.1365-2362.2002.01063.x

48. Ishimura E, Okuno S, Tsuboniwa N, Norimine K, Fukumoto S, Yamakawa $\mathrm{K}$, et al. Significant positive association between parathyroid hormone and fat mass and lean mass in chronic hemodialysis patients. J Clin Endocrinol Metab. (2013) 98:1264-70. doi: 10.1210/jc.2012-3883

49. Shinaberger CS, Kopple JD, Kovesdy CP, McAllister CJ, van Wyck D, Greenland S, et al. Ratio of paricalcitol dosage to serum parathyroid hormone level and survival in maintenance hemodialysis patients. Clin J Am Soc Nephrol. (2008) 3:1769-76. doi: 10.2215/CJN.01760408

50. Molina P, Carrero JJ, Bover J, Chauveau P, Mazzaferro S, Torres PU, et al. Vitamin D, a modulator of musculoskeletal health in chronic kidney disease. $J$ Cachexia Sarcopenia Muscle. (2017) 8:686-701. doi: 10.1002/jcsm.12218

51. Cheung WW, Ding W, Hoffman HM, Wang Z, Hao S, Zheng R, et al. Vitamin $\mathrm{D}$ ameliorates adipose browning in chronic kidney disease cachexia. Sci Rep. (2020) 10:14175. doi: 10.1038/s41598-020-70190-z

52. Bacchetta J, Schmitt CP, Ariceta G, Bakkaloglu SA, Groothoff J, Wan M, et al. Cinacalcet use in paediatric dialysis: a position statement from the European Society for Paediatric Nephrology and the chronic kidney disease-mineral and bone disorders working group of the ERA-EDTA. Nephrol Dial Transplant. (2020) 35:47-64. doi: 10.1093/ndt/gfz159

53. Greenbaum LA, Jeck N, Klaus G, Fila M, Stoica C, Fathallah-Shaykh $S$, et al. Safety and efficacy of sucroferric oxyhydroxide in pediatric patients with chronic kidney disease. Pediatr Nephrol. (2021) 36:12331244. doi: 10.1007/s00467-020-04805-y

Conflict of Interest: The authors declare that the research was conducted in the absence of any commercial or financial relationships that could be construed as a potential conflict of interest.

Publisher's Note: All claims expressed in this article are solely those of the authors and do not necessarily represent those of their affiliated organizations, or those of the publisher, the editors and the reviewers. Any product that may be evaluated in this article, or claim that may be made by its manufacturer, is not guaranteed or endorsed by the publisher.

Copyright $\odot 2021$ Karava, Kondou, Dotis, Christoforidis, Taparkou, Tsioni, Farmaki, Kollios, Siomou, Liakopoulos and Printza. This is an open-access article distributed under the terms of the Creative Commons Attribution License (CC BY). The use, distribution or reproduction in other forums is permitted, provided the original author(s) and the copyright owner(s) are credited and that the original publication in this journal is cited, in accordance with accepted academic practice. No use, distribution or reproduction is permitted which does not comply with these terms. 